\title{
Jubilee of Atomic Emission Analysis
}

\author{
DOI: $10.1134 / \mathrm{S} 1061934809040017$
}

The possibilities of analytical chemistry are increasing rapidly both because of the development of new methods and directions and as a result of the sophistication of the known, currently-used methods and wellestablished directions. About fifteen years ago only a handful of people had heard about ion mobility spectrometry, and now it is one of the main methods for the detection of explosive substances. Twenty years ago there was no liquid chromatography mass spectrometry, and nowadays this method is used to solve many problems in molecular biology and neighboring fields. Mass spectrometry as a whole had long been an expensive and elaborate method used primarily in scientific research and unique elemental analyses. Now, about a quarter of the papers in the journal Analytical Chemistry in some way relate to mass spectrometry, and moreover, they relate to organic mass spectrometry with an electron spray or MALDI ionization rather than to ICP-MS. Until recently the direction, which is now called substance analysis, virtually did not exist (only phase analysis of rocks, minerals, and metals). Today the development and use of techniques for the detection and quantitative determination of individual chemical and physical forms of components is attracting increasing attention. The use of mathematical methods in chemical analysis, which began with mathematical statistics, has grown in a separate direction, chemometrics.

Among the rapidly developing applications of chemical analytical methods and means, applications in medical diagnostics (for example, the detection of the early stages of oncological diseases by determining changed nucleotides in urine or by the composition of exhaled air) and the control of foodstuffs make the strongest impression. The certification of almost all products rarely goes without the determination of their chemical composition.

The progress in the development of analytical methods can be assessed through an example of atomic emission analysis. In 2009, atomic emission analysis will be 150 years old. This method was a product of the collaboration of the physicist Kirchhoff and the chemist Bunsen, a product of, possibly, the first such collaboration, at least amongst the well-known ones collaborations whose results have endured over time. This collaboration amongst the scientists from Heidelberg was a remarkable example, even a symbol: over one and a half centuries, the progress and applications of the method were the result of joint efforts of physicists and chemists.

In these 150 years, various excitation sources were created, from flame to spark, arc, two-jet plasmatron, and inductively coupled plasma. Spectral decomposition with prisms was successfully replaced by decomposition with diffraction lattices with increasing numbers of lines per unit length; other monochromatization techniques have also been proposed. Spectrum registration was also improved: from visual to photographic, then to photoelectric. The processing of primary spectral data by mathematical methods based on computers has played an important role. Numerous and quite significant applied problems of analysis and analytical control are being solved because of the commercial production of good instruments, the manufacture of reference samples, the availability of mathematical programs, and, not least, thanks to the abundant experience accumulated, also in Russia.

Speaking about Russia, we refer not only to practical experience, which is finally no more than the derivative from something more important. Of significant scientific value were the works of Russian scientists, Sventitskii, Striganov, Prokof'ev, Raiskii, Zaidel', Neddler, Rusanov, M.E. Britske, and the efforts of the Commission on Spectroscopy of the Academy of Sciences of the USSR headed by G.S. Landsberg and subsequently S.L. Mandel'shtam.

An important role was and is played by the equipment of spectrometers with multichannel analyzers of emission spectra based on diode arrays. MAES analyzers designed in Novosibirsk occupy a significant place here. The good design, wide introduction, and successful practical use of these analyzers are the result of the originality of the approaches used, the high qualification of the developers, and the dedication and enthusiasm of the managers and employees of the VMK Optoelectronics Company. This determines the prestige of the company and the attractiveness of the workshops and symposia it organizes. 